\title{
GENERALIZATIONS OF SEMI-FREDHOLM OPERATORS
}

\author{
RICHARD BOULDIN
}

(Communicated by Palle E. T. Jorgensen)

\begin{abstract}
On nonseparable Hilbert spaces there are multiple sets of operators that are analogous to the semi-Fredholm operators on a separable space. We develop the properties of these sets and relate those properties to some recent research. We conclude with a theorem that indicates precisely how far one can go from a given generalized semi-Fredholm operator (or generalized Fredholm operator) and retain the property of generalized semi-Fredholmness (or generalized Fredholmness).
\end{abstract}

\section{INTRODUCTION}

By "operator" we mean a bounded linear operator $T$ defined on a Hilbert space $H$; we do not assume $H$ to be separable. Let $\mathscr{B}(H)$ denote the operators on $H$. When $H$ is not separable there are multiple sets of operators that might be used as natural generalizations of the Fredholm operators on a separable Hilbert space. See Definition 2.7 and Theorem 2.8 of [11], and the references listed there. Much less attention has been given to the semi-Fredholm operators. This paper continues our study of nonseparable Hilbert spaces ([5], [6], [7]) by determining properties of several classes of operators analogous to the semiFredholm operators on a separable space.

We shall use some concepts from earlier papers. Let $U|T|$ be the usual polar factorization of $T$, and let $E(\cdot)$ be the spectral measure for the nonnegative operator $|T|$. In [5] we defined ess nul $T$ by the equation

$$
\text { ess null } T=\inf \{(\operatorname{dim} E([0, \varepsilon)) H: \varepsilon>0\} \text {, }
$$

and by definition ess def $T$ is ess nul $T^{*}$. In [5] we showed that the closure of the invertible operators is the set of operators $T$ such that ess nul $T=$ ess def $T$.

In [7] we defined the modulus of invertibility, denoted $\rho(T)$, by the equation

$$
\rho(T)=\inf \{\lambda: \operatorname{dim} E((\lambda-\varepsilon, \lambda+\varepsilon)) H=\operatorname{dim} H \text { for } \varepsilon>0\} .
$$

This quantity is related to the distance between $T$ and the invertible operators. It follows from Theorem 4 in [8] that $\rho(T)$ is precisely the distance from $T$

Received by the editors May 27, 1994.

1991 Mathematics Subject Classification. Primary 47A58, 47A53; Secondary 47A05.

Key words and phrases. Semi-Fredholm operator, nonseparable Hilbert space, essential nullity, modulus of invertibility, stability theorem. 
to the set $\{A$ : ess nul $A=0\}$. In this paper we use a natural generalization of $\rho(T)$ defined in $\S 2$.

Recall that the minimum modulus of $T$, denote $m(T)$, is defined to be $\inf \{\|T f\|:\|f\|=1\}$. The essential spectrum of $T$, denoted $\sigma_{e}(T)$, is the set $\{z:(T-z I)$ is not Fredholm $\}$, and the essential minimum modulus $m_{e}(T)$ is defined to be $\inf \left\{\lambda: \lambda \in \sigma_{e}(|T|)\right\}$. In [2] we showed that $m_{e}(T)$ was the minimum modulus of the projection of $T$ into the Calkin algebra, which is defined in the next section. In [2] we computed the distance from $T$ to the invertible operators in terms of $m_{e}(T)$. In this paper we shall use a generalization of $m_{e}(T)$.

We shall use the following standard notation. The closure of a set $\mathscr{S}$ is denoted $\mathscr{S}^{-}$. The restriction of $T$ to a subspace $H_{0}$ is denoted $T \mid H_{0}$.

\section{Preliminary Results}

We begin by defining a set of operators that form a closed two-sided ideal for the ring of operators $\mathscr{B}(H)$. We say that $A$ belongs to $\mathscr{I}_{\beta}$ provided that any (closed) subspace $K$ contained in the range of $A$, denoted $A H$, has the property that $\operatorname{dim} K<\beta$. The next theorem will relate the preceding definition to other definitions that have appeared in the literature.

Theorem 1. The following are equivalent.

(i) $A \in \mathscr{I}_{\beta}$.

(ii) There is a sequence $\left\{A_{k}\right\} \subset \mathscr{B}(H)$ such that $\left\|A-A_{k}\right\| \rightarrow 0$ as $k \rightarrow \infty$ and $\operatorname{dim}\left(A_{k} H\right)^{-}<\beta$.

(iii) For each positive $\varepsilon$ there is a subspace $H_{\varepsilon}$ such that $\left\|A \mid H_{\varepsilon}\right\|<\varepsilon$ and $\operatorname{dim}\left(H_{\varepsilon}\right)^{\perp}<\beta$.

Proof. (i) $\Rightarrow$ (ii) Let $U|A|$ be the usual polar factorization of $A$, and let $E(\cdot)$ be the spectral measure for $|A|$. Let a positive $\varepsilon$ be given, and let $H_{\varepsilon}$ denote $E([\varepsilon, \infty)) H$. Since $|A|$ is obviously bounded below on $H_{\varepsilon}$ and $U$ is isometric on $(|A| H)^{-}$, we see that $|A| H_{\varepsilon}$ and $A H_{\varepsilon}=U|A| H_{\varepsilon}$ are both closed subspaces. Clearly $A H_{\varepsilon}$ is contained in $A H$, and, consequently, $\operatorname{dim} A H_{\varepsilon}<\beta$.

Define the operator $A_{\varepsilon}$ to agree with $A$ on $H_{\varepsilon}$ and to agree with 0 on $\left(H_{\varepsilon}\right)^{\perp}$. Clearly $\operatorname{dim} A_{\varepsilon} H<\beta$, and

$$
\begin{aligned}
\left\|A-A_{\varepsilon}\right\| & =\left\|\left(A-A_{\varepsilon}\right) \mid\left(H_{\varepsilon}\right)^{\perp}\right\| \\
& =\left\|A\left|\left(H_{\varepsilon}\right)^{\perp}\|=\|\right|\right\| A||\left(H_{\varepsilon}\right)^{\perp} \| \leq \varepsilon .
\end{aligned}
$$

It is clear that (ii) holds.

(ii) $\Rightarrow$ (iii) Let positive $\varepsilon$ be given, and choose $k$ such that $\left\|A-A_{k}\right\|<\varepsilon$ with $\operatorname{dim}\left(A_{k} H\right)^{-}<\beta$. Let $K_{\varepsilon}=\left(\left(A_{k}\right)^{*} H\right)^{-}$, and note that $A_{k}$ is zero on $\left(K_{\varepsilon}\right)^{\perp}$. Clearly, $\left\|A \mid\left(K_{\varepsilon}\right)^{\perp}\right\|<\varepsilon$ holds. Let $H_{\varepsilon}=\left(K_{\varepsilon}\right)^{\perp}$, and note that

$$
\operatorname{dim}\left(H_{\varepsilon}\right)^{\perp}=\operatorname{dim} K_{\varepsilon}=\operatorname{dim}\left(A_{k} H\right)<\beta .
$$

(iii) $\Rightarrow$ (i) Let $K$ be a (closed) subspace contained in $A H$, and let $A^{+}$be the inverse of $A \mid\left(A^{*} H\right)^{-}$. Let $H_{0}=A^{+} K$, and note that $A \mid H_{0}$ maps $H_{0}$ onto $K$ in a one-to-one way. Thus, $H_{0}$ and $K$ have the same dimension, and there is a positive $\varepsilon$ such that

$$
\|A f\| \geq 2 \varepsilon\|f\| \text { for } f \in H_{0} .
$$


By hypothesis there is a subspace $H_{\varepsilon}$ such that $\left\|A \mid H_{\varepsilon}\right\|<\varepsilon$ and $\operatorname{dim}\left(H_{\varepsilon}\right)^{\perp}<$ $\beta$. Let $P$ be the projection onto $\left(H_{\varepsilon}\right)^{\perp}$. If $f$ is a nonzero vector belonging to $\operatorname{ker} P \mid H_{0}$ then $f=(I-P) f \in H_{\varepsilon}$ and $\|A f\| \leq \varepsilon\|f\|$. This contradicts the construction of $H_{0}$, and so we conclude that $P \mid H_{0}$ is one-to-one. It follows that

$$
\operatorname{dim} K=\operatorname{dim} H_{0}=\operatorname{dim} P H_{0} \leq \operatorname{dim}\left(H_{\varepsilon}\right)^{\perp}<\beta,
$$

which verifies $(\mathrm{i})$.

We need a modest generalization of the modules of invertibility, which was defined in [7]. Let $\beta$ be an infinite cardinal number that does not exceed $\operatorname{dim} H$. Define $\rho_{\beta}(T)$ by the equation

$$
\rho_{\beta}(T)=\inf \{\lambda: \operatorname{dim} E((\lambda-\delta, \lambda+\delta)) H \geq \beta \text { for } \delta>0\}
$$

where $E(\cdot)$ is the spectral measure for $|T|=\left(T^{*} T\right)^{1 / 2}$. Consideration of the function $u(x)=\operatorname{dim} E([0, x)) H$ shows that the set of $\lambda$ above cannot be empty. It is clear that $\gamma \geq \beta$ implies $\rho_{\gamma}(T) \geq \rho_{\beta}(T)$.

The first part of the next result is well known (see [11]); the rest of it is new.

Theorem 2. $\mathscr{I}_{\beta}$ is a closed two-sided ideal of $\mathscr{B}(H)$, and $\mathscr{B}(H) / \mathscr{I}_{\beta}$ is a $C^{*}$-algebra. If $m_{\beta}(T)$ denotes the minimum modulus of the image of $T$ in $\mathscr{B}(H) / \mathscr{I}_{\beta}$, denoted $[T]$, then $m_{\beta}(T)>0$ if and only if $[T]$ is left-invertible. Furthermore, $m_{\beta}(T)=\rho_{\beta}(T)$.

Proof. It is routine to show that $\mathscr{J}_{\beta}$ is a closed two-sided ideal; see Theorem 0 of [11]. It follows from Theorem 5.38 of [10] that $\beta(H) / \mathscr{F}_{\beta}$ is a $C^{*}$-algebra provided the involution $[T]^{*}$ is defined to be $\left[T^{*}\right]$. Let this $C^{*}$-algebra be represented as an algebra of operators. Thus, $m_{\beta}(T)$ is meaningful. Since the square root is the limit of polynomials, we have

$$
|[T]|=\left([T]^{*}[T]\right)^{1 / 2}=\left(\left[T^{*} T\right]\right)^{1 / 2}=\left[\left(T^{*} T\right)^{1 / 2}\right]=[|T|] .
$$

If $\sigma(A)$ denotes the spectrum of $A$, then it is elementary to see that

$$
m(A)=\inf \{\lambda: \lambda \in \sigma(|A|)\}
$$

and, thus,

$$
m_{\beta}(T)=\inf \{\lambda: \lambda \in \sigma([|T|]) .
$$

Suppose $[B]$ is a left inverse for $[T]$. For the sake of a contradiction assume that $m_{\beta}(T)=0$ and choose a sequence of unit vectors $\left\{g_{1}, g_{2}, \ldots\right\}$ from the space $K$ on which $\mathscr{B}(H) / \mathscr{I}_{\beta}$ is represented such that $\left\|[T] g_{k}\right\| \rightarrow 0$. Since

$$
1=\left\|g_{k}\right\|=\left\|[B][T] g_{k}\right\| \leq\|[B]\|\left\|[T] g_{k}\right\|
$$

holds, we have a contradiction. Thus, $m_{\beta}(T)$ is positive provided $[T]$ is leftinvertible.

In order to prove the converse we assume that $m_{\beta}(T)>0$. Choose $\varepsilon_{0}$ such that $0<\varepsilon<2 \varepsilon_{0}$ implies that $\operatorname{dim} E([0, \varepsilon)) H=$ ess nul $T$. Since $[E([0, \varepsilon))]$ is a nonnegative idempotent, it is a projection. If $g$ is a unit vector in the range of $[E([0, \varepsilon))]$, then

$$
\varepsilon>\|[T][E([0, \varepsilon))] g\| \geq m_{\beta}(T),
$$

which implies that no such unit vector exists. Thus, $\operatorname{dim} E([0, \varepsilon)) H=\operatorname{ess} \operatorname{nul} T$ is less than $\beta$. 
Define $R$ on $E\left(\left(\varepsilon_{0}, \infty\right)\right) H$ to be the inverse of $|T| \mid E\left(\left(\varepsilon_{0}, \infty\right)\right) H$ and on $E\left(\left[0, \varepsilon_{0}\right]\right) H$ let it be zero. The preceding paragraph implies that $\left[E\left(\left[0, \varepsilon_{0}\right]\right)\right]=$ $[0]$ or $\left[E\left(\left(\varepsilon_{0}, \infty\right)\right)\right]=[I]$. Since

$$
\left(R U^{*}\right) T=R U^{*} U|T|=R|T|=E\left(\left(\varepsilon_{0}, \infty\right)\right),
$$

we see that $[T]$ is left-invertible.

Now we direct our attention to proving that $m_{\beta}(T)=\rho_{\beta}(T)$. First, we recall a useful fact from Theorem 2.6 of [11]. The coset [T] is left-invertible if and only if $T$ is bounded below on some subspace with codimension less than $\beta$.

Consider the case that $m_{\beta}(T)=0$. So [T] is not left-invertible. If $\varepsilon>0$ and $E(\cdot)$ is the spectral measure for $|T|$, then $|T|$ is bounded below on $E((\varepsilon, \infty)) H$. According to the preceding paragraph, the codimension of $E((\varepsilon, \infty)) H$, which is $\operatorname{dim} E([0, \varepsilon]) H$, must not be less than $\beta$. It follows from

$$
\operatorname{dim} E([0, \varepsilon]) H \geq \beta
$$

and the arbitrariness of $\varepsilon$ that $\rho_{\beta}(T)=0$.

Conversely, assume that $\rho_{\beta}(|T|)=0$ and note that $\operatorname{dim} E([0, \varepsilon]) H \geq \beta$ for all positive $\varepsilon$ sufficiently small. If $m_{\beta}(T)$ were positive, then there would be a subspace $H_{0}$ such that $T \mid H_{0}$ is bounded below and $\operatorname{codim} H_{0}<\beta$. If $Q$ is the orthogonal projection onto $\left(H_{0}\right)^{\perp}$ and $\varepsilon$ is a positive number such that $\|T f\| \geq 2 \varepsilon\|f\|$ for $f \in H_{0}$, then the restriction of $Q$ to $E([0, \varepsilon]) H$ must have nontrivial kernel. Suppose $g \in E([0, \varepsilon]) H$ and $Q g=0$. Since $g \in H_{0} \cap E([0, \varepsilon]) H$, we have a contradiction; this proves that no subspace such as $H_{0}$ exists. Hence, $m_{\beta}(T)=0$, and we have proved that $m_{\beta}(T)=0$ if and only if $\rho_{\beta}(T)=0$.

For the sake of a contradiction suppose $m_{\beta}(T)$ and $\rho_{\beta}(T)$ are positive and

$$
m_{\beta}(T)=\lambda<\rho_{\beta}(T) \text {. }
$$

Then $m_{\beta}(|T|-\lambda)=0$ while $\rho_{\beta}(|T|-\lambda)>0$, which is a contradiction. A similar argument dismisses the opposite inequality, and we have proved that $m_{\beta}(T)=\rho_{\beta}(T)$.

If $\beta=\aleph_{0}$, then it is well known that $\mathscr{I}_{\beta}$ is the set of compact operators, and $\mathscr{B}(H) / \mathscr{I}_{\beta}$ is the usual Calkin algebra. In that case the notation $m_{\beta}(T)$ is replaced with the notation $m_{e}(T)$ for the essential minimum modulus, which was studied in [2]. Of course, in that case $\rho_{\beta}(T)$ is the modulus of invertibility $\rho(T)$.

\section{BASIC RESULTS}

Let $\beta$ be an infinite cardinal number that does not exceed $\operatorname{dim} H$. We say that $A$ is $\beta$-left-invertible provided there is an operator $B$ such that $(I-B A)$ belongs to $\mathscr{I}_{\beta}$. Define $\beta$-right-invertible analogously. We say that $A$ is $\beta$ semi-invertible if it has at least one of the two preceding properties and that it is $\beta$-Fredholm if it has both properties.

If $H$ is a separable Hilbert space and $\beta=\operatorname{dim} H$, then the preceding concepts reduce to "left-semi-Fredholm", and "right-semi-Fredholm", "semiFredholm", and "Fredholm", respectively. A strong argument can be made that the generalization that is most analogous to the original concept is obtained by setting $\beta=\operatorname{dim} H$ in the case that $H$ is non-separable. 
We can give some enlightening characterizations of the previously defined concepts.

Theorem 3. The following are equivalent.

(i) $T$ is $\beta$-left-ivnertible.

(ii) $\rho_{\beta}(T)>0$.

(iii) ess nul $T<\beta$.

(iv) For all positive $\varepsilon$ sufficiently small the inequality $\left\|T \mid H_{\varepsilon}\right\|<\varepsilon$ implies that $\operatorname{dim} H_{\varepsilon}<\beta$.

Proof. (i) $\Rightarrow$ (ii) This follows from Theorem 2.

(ii) $\Rightarrow$ (iii) For brevity let $\tau=\rho_{\beta}(T)$ and choose $\lambda$ to satisfy the inequalities $0 \leq \lambda<\tau$. For each $\lambda$ there is a positive number $\delta_{\lambda}$ such that

$$
\operatorname{dim} E\left(\left(\lambda-\delta_{\lambda}, \lambda+\delta_{\lambda}\right)\right) H<\beta .
$$

Choose positive $\varepsilon$ sufficiently small that $\operatorname{dim} E([0, \varepsilon)) H=$ ess nul $T$ and $\varepsilon<\tau$; note that $\left\{\left(\lambda-\delta_{\lambda}, \lambda+\delta_{\lambda}\right): 0 \leq \lambda<\tau\right\}$ is an open cover of $[0, \varepsilon]$. Let $\left\{\left(\lambda_{1}-\delta_{1}, \lambda_{1}+\delta_{1}\right), \ldots,\left(\lambda_{n}-\delta_{n}, \lambda_{n}+\delta_{n}\right)\right\}$ be a finite subcover. We note that

$$
\begin{aligned}
\operatorname{dim} E([0, \varepsilon)) H & \leq \sum_{j=1}^{n} \operatorname{dim} E\left(\left(\lambda_{j}-\delta_{j}, \lambda_{j}+\delta_{j}\right)\right) H \\
& <n \beta=\beta .
\end{aligned}
$$

Since $\varepsilon$ was arbitrarily small, it follows that ess nul $T<\beta$.

(iii) $\Rightarrow$ (iv) Choose positive $\varepsilon$ sufficiently small that $\operatorname{dim} E([0, \varepsilon)) H=$ ess nul $T$. By Lemma 4 of [5] it follows that $\left\|T \mid H_{\varepsilon}\right\|<\varepsilon$ implies that $\operatorname{dim} H_{\varepsilon} \leq$ ess nul $T<\beta$.

(iv) $\Rightarrow$ (i) Theorem 2 showed the equivalence " $T$ is $\beta$-left-invertible" and " $m_{\beta}(T)>0$ "; the final conclusion of Theorem 2 shows that this is equivalent to " $\rho_{\beta}(T)>0$ ". Thus, it suffices to show that (iv) implies $\rho_{\beta}(T)>0$.

Let $\varepsilon$ be a positive number such as promised in (iv), and let $E(\cdot)$ be the spectral measure for $|T|$. Let $H_{\varepsilon}=E([0, \varepsilon / 2]) H$, and note that $\left\|T \mid H_{\varepsilon}\right\|<\varepsilon$. Thus, we know that $\operatorname{dim} H_{\varepsilon}<\beta$. It follows that we can choose positive $\delta$ for any $\lambda$ satisfying $0 \leq \lambda<\varepsilon / 2$ such that $\operatorname{dim} E((\lambda-\delta, \lambda+\delta)) H \leq \operatorname{dim} H_{\varepsilon}<\beta$. It is now clear that $\rho_{\beta}(T) \geq \varepsilon / 2$, which concludes the proof.

We omit the proof of the next theorem since it can be proved by analogy to the proof of Theorem 3 or deduced as a consequence of that theorem.

Theorem 4. The following are equivalent.

(i) $T$ is $\beta$-right-invertible.

(ii) $\rho_{\beta}\left(T^{*}\right)>0$.

(iii) ess def $T<\beta$.

(iv) For all positive $\varepsilon$ sufficiently small the inequality $\left\|T^{*} \mid H_{\varepsilon}\right\|<\varepsilon$ implies that $\operatorname{dim} H_{\varepsilon}<\beta$.

The next theorem follows from Theorems 3 and 4. Part (iii) of the next theorem gives a particularly simple characterization of " $\beta$-Fredholm".

Theorem 5. The following are equivalent.

(i) $T$ is $\beta$-Fredholm.

(ii) $\min \left\{\rho_{\beta}(T), \rho_{\beta}\left(T^{*}\right)\right\}>0$. 
(iii) $\max \{$ ess nul $T$, ess def $T\}<\beta$.

(iv) For all positive $\varepsilon$ sufficiently small either of the inequalities $\left\|T \mid H_{\varepsilon}\right\|<\varepsilon$ or $\left\|T^{*} \mid H_{\varepsilon}\right\|<\varepsilon$ implies that $\operatorname{dim} H_{\varepsilon}<\beta$.

If $\rho_{\beta}(T)$ and $\rho_{\beta}\left(T^{*}\right)$ are both positive, then they are equal. This follows from the fact that $\rho_{\beta}(A)=m_{\beta}(A)$, proved in Theorem 2, and the argument used to prove part (vii) of Theorem 2 of [2].

Let $\mathscr{S}_{\beta}$ denote the $\beta$-semi-invertible operators in $\mathscr{B}(H)$. The next theorem establishes some properties analogous to familiar properties of the semiFredholm operators.

Theorem 6. (i) $\mathscr{S}_{\beta}$ is open in operator norm.

(ii) $\mathscr{S}_{\beta}$ is dense in $\mathscr{B}(H)$.

(iii) If every subspace of $K H$ has dimension less than $\beta$ and $A \in \mathscr{S}_{\beta}$, then $(A+K) \in \mathscr{S}_{\beta}$.

(iv) If $A$ and $B$ are $\beta$-left-invertible ( $\beta$-right-invertible), then $A B$ is the same.

(v) If $A \in \mathscr{S}_{\beta}$ and $B$ is $\beta$-Fredholm, then $A B, B A \in \mathscr{S}_{\beta}$.

(vi) If $B$ is invertible and $A \in \mathscr{S}_{\beta}$, then $B A B^{-1} \in \mathscr{S}_{\beta}$.

Proof. (i) In view of Theorems 3 and 4 , for each $T \in \mathscr{S}_{\beta}$ we have either $\rho_{\beta}(T)>0$ or $\rho_{\beta}\left(T^{*}\right)>0$. For a representative case we assume the former. Recalling the notation and conventions in Theorem 2 and its proof, we note that $m_{\beta}(T)>0$. Let $\delta=m_{\beta}(T)$, and choose $A$ such that $\|T-A\|<\delta$.

Let $g$ be any unit vector in the Hilbert space on which $\mathscr{B}(H) / \mathscr{I}_{\beta}$ is represented. In the notation of the proof of Theorem 2 we have

$$
\|[A] g\| \geq\|[T] g\|-\|[T-A] g\| \geq \delta-\|T-A\|>0 .
$$

We may conclude that

$$
\rho_{\beta}(A)=m_{\beta}(A) \geq \delta-\|T-A\|>0 .
$$

It follows that $A \in \mathscr{S}_{\beta}$ and that $\mathscr{S}_{\beta}$ is open.

(ii) It suffices to approximate a given $T \notin \mathscr{S}_{\beta}$. Let positive $\varepsilon$ be given, and let $T=U|T|$ be the usual polar factorization of $T$. Let $E(\cdot)$ be the spectral measure for $|T|$; define $R$ to coincide with $|T|$ on $E([\varepsilon / 3, \infty)) H$, and let it coincide with $(\varepsilon / 3) I$ on $E([0, \varepsilon / 3)) H$. Note that

$$
\|R f\| \geq(\varepsilon / 3)\|f\| \text { for } f \in H .
$$

Define $A$ to be $U R$ on $E((0, \infty)) H$ and $R$ on $E(\{0\}) H$; note that it is left-invertible since it is bounded below. Since we have

$$
\|T-A\| \leq\||T|-R\|=\|(|T|-R) \mid E([0, \varepsilon / 3)) H\|<\varepsilon,
$$

we see that $T \in\left(\mathscr{S}_{\beta}\right)^{-}$, as desired.

(iii) Since $[A+K]=[A]$, we see that $m_{\beta}(A+K)=m_{\beta}(A)$ and this follows from Theorems 2,3 , and 4.

(iv) If $D$ and $C$ are $\beta$-left-inverses for $A$ and $B$, respectively, then $C D$ is a $\beta$-left-inverse for $A B$.

(v) This follows from (iv) since $B$ has both a $\beta$-left-inverse and a $\beta$-rightinverse. 
(vi) If $C$ is a $\beta$-left-inverse for $A$, then $B C B^{-1}$ is a $\beta$-left-inverse for $B A B^{-1}$.

\section{A STABILITY THEOREM}

Now we are prepared to prove the kind of stability theorem that has been significant in perturbation theory.

Theorem 7. (i) If $\delta_{1}=\rho_{\beta}(T)$ and $\|T-A\|<\delta_{1}$, then $A$ is $\beta$-left-inertible.

(ii) If $\delta_{2}=\rho_{\beta}\left(T^{*}\right)$ and $\|T-A\|<\delta_{2}$, then $A$ is $\beta$-right-invertible.

(iii) If $\delta=\min \left\{\delta_{1}, \delta_{2}\right\}$ and $\|T-A\|<\delta$, then $A$ is $\beta$-Fredholm.

(iv) Each of the above statements is false if the $\delta$ constant is replaced with a larger number.

Proof. The proof of part (i) of Theorem 6 actually proved part (i) above. To prove (ii) apply (i) to $T^{*}$ and $A^{*}$. Part (iii) follows from (i) and (ii).

In order to prove (iv) we assume that a positive $\varepsilon$ is given. Let $E($ ) represent the spectral measure for $|T|$ where $T=U|T|$ is the usual polar factorization. Let $Q$ denote the projection $E\left(\left[0, \varepsilon+\rho_{\beta}(T)\right)\right)$, and let $H_{\varepsilon}$ denote $Q H$. By the definition of $\rho_{\beta}(T)$ we know that $\operatorname{dim} H_{\varepsilon} \geq \beta$. Define $B$ to coincide with $T$ on $\left(H_{\varepsilon}\right)^{\perp}$ and to be zero on $H_{\varepsilon}$; so $B=T(I-Q)$. Note that

$$
\|T-B\|=\left\|T \mid H_{\varepsilon}\right\| \leq \varepsilon+\rho_{\gamma}(T) .
$$

Since

$$
\text { ess nul } B=\operatorname{nul} B=\operatorname{dim} H_{\varepsilon} \geq \beta,
$$

we see that $B$ is not $\beta$-left-invertible. This proves that $\delta_{1}$ is the largest constant that makes (i) true.

Applying the construction in the preceding paragraph to $T^{*}$ shows that $\delta_{2}$ is the largest constant that makes (ii) true. It follows that replacing $\delta$ in part (iv) with a larger constant will result in the loss of either " $\beta$-left-invertible" or " $\beta$-right-invertible", which means that " $\beta$-Fredholm" is lost.

If one chooses $\beta$ in Theorem 7 to be $\aleph_{0}$, so that $\mathcal{I}_{\beta}$ consists of the compact operators, then part (i) of the previous theorem gives the distance from a leftsemi-Fredholm operator to the set of operators that are not left-semi-Fredholm. If $\beta=\aleph_{0}$, then part (ii) describes the distance from a right-semi-Fredholm operator to the set of operators that are not right-semi-Fredholm, and part (iii) computes the distance from a Fredholm operator to the complement of the Fredholm operators.

\section{REFERENCES}

1. C. Apostol, L. A. Fialkow, D. A. Herrero, and D. Voiculescu, Approximation of Hilbert space operators, Vol. II, Pitman, Boston, 1984.

2. R. H. Bouldin, The essential minimum modulus, Indiana Univ. Math. J. 30 (1981), 513-517.

3. Approximation by operators with fixed nullity, Proc. Amer. Math. Soc. 103 (1988), 141-144.

4. $\ldots$, The distance to operators with a fixed index, Acta Sci. Math. (Szeged) 54 (1990), 139-143.

5. $\ldots$, Closure of invertible operators on a Hilbert space, Proc. Amer. Math. Soc. 108 (1990), 721-726. 
6. Approximating Fredholm operators on a nonseparable Hilbert space, Glasgow Math. J. 35 (1993), 167-178.

7. (1992), 489-497.

8. __ Largely singular operators, J. Math. Anal. Appl. 188 (1994), 141-150.

9. L. Burlando, Distance formulas on operators whose kernel has fixed Hilbert dimension, Rend. Mat. (7) 10 (1990), 209-238.

10. R. G. Douglas, Banach algebra techniques in operator theory, Academic Press, New York, 1972.

11. G. Edgar, J. Ernest, and S. G. Lee, Weighing operator spectra, Indiana Univ. Math. J. 121 (1971), 61-80.

12. R. Harte, Regular boundary elements, Proc. Amer. Math. Soc. 99 (1987), 328-330.

13. D. A. Herrero, Approximation of Hilbert space operators, Vol. I, Pitman, Boston, 1982.

Department of Mathematics, University of Georgia, Athens, Georgia 30602 